\title{
Multiband optical polarimetry of BL Lacertae objects with the Nordic Optical Telescope ${ }^{\star, \star \star}$
}

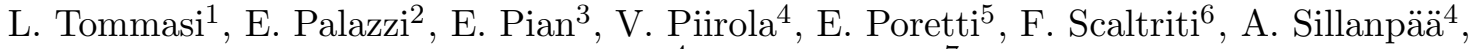 \\ L. Takalo ${ }^{4}$, and A. Treves ${ }^{7}$
}

1 Dipartimento di Fisica, Università di Milano, 20133 Milano, Italy

2 ITESRE-CNR, 40129 Bologna, Italy

3 Osservatorio Astronomico di Trieste, 34131 Trieste, Italy (also ITESRE-CNR, 40129 Bologna, Italy)

4 Tuorla Observatory, 21500 Piikkio, Finland

5 Osservatorio Astronomico di Brera, 23807 Merate, Italy

6 Osservatorio Astronomico di Torino, 10025 Pino Torinese, Italy

7 Dipartimento di Scienze, Università dell'Insubria, 22100 Como, Italy

Received 3 January 2001 / Accepted 8 June 2001

\begin{abstract}
Optical polarization of seven selected BL Lac objects in $U B V R I$ bands was studied with the Nordic Optical Telescope from December 10-14, 1999. Two of them, 3C 66A and PKS 0735+178, were monitored for 4 nights for a total integration time of 4.75 and 5.5 hours, respectively. Other objects (1 Jy 0138-097, H 0414+009, PKS 0823-223, OJ287 and BL Lac) were observed sparsely during the run. Apart from PKS 0823-223 (more polarized than observed in the past), the sources show levels of flux and polarization consistent with results at previous epochs. 3C 66A and PKS 0735+178 were intensively observed during December 11 and 12 and exhibited variability of polarization, both on internight and intranight time scales. Wavelength dependence of polarization has been investigated, as well as circular polarization. The results are discussed within the standard model for BL Lacs.
\end{abstract}

Key words. polarization - radiation mechanisms: non-thermal - galaxies: active - BL Lacertae objects: general BL Lacertae objects: individual: 3C 66A - BL Lacertae objects: individual: PKS 0735+178

\section{Introduction}

Synchrotron radiation from a relativistic jet pointing toward the observer is thought to produce the emission of BL Lac objects, from radio up to X-rays and sometimes soft gamma rays. The maximum power output of the synchrotron component is located at different energies in different objects. In particular, following Padovani \& Giommi (1995), BL Lacs can be divided into two classes: Low energy cutoff BL Lacs (LBL) and High energy cutoff BL Lacs (HBL). In the former, the synchrotron peak, in a $\nu F_{\nu}$ representation, lies in the optical/infrared domain, while in the latter it occurs in the UV/X-ray band. A second, higher energy peak, occurring in the $\mathrm{GeV} / \mathrm{TeV}$

Send offprint requests to: E. Pian, e-mail: pian@ts.astro.it

* Based on observations made with the Nordic Optical Telescope, operated on the island of La Palma jointly by Denmark, Finland, Iceland, Norway, and Sweden, in the Spanish Observatorio del Roque de los Muchachos of the Instituto de Astrofisica de Canarias.

** Table 2 is only available in electronic form at http://www .edpsciences.org domain for LBL/HBL, respectively, is due to inverse Compton radiation. The strongest observational evidence in favour of synchrotron radiation is the high polarization recorded in all the bands from radio to UV (Brindle et al. 1986; Saikia \& Salter 1988; Allen et al. 1993; Gabuzda et al. 1996). This is related to the presence of a regular (at least in one of its components) magnetic field. The relativistic beaming amplifies the radiation from the nucleus with respect to the unpolarized light coming from the host galaxy and the polarization can be as high as $30 \%$. Only a few objects have been observed up to now in search for polarization variability on intranight timescales (Puschell et al. 1979; Moore et al. 1987; Impey et al. 2000; Tommasi et al. 2001b).

Data presented in this paper have been collected with the Nordic Optical Telescope (NOT) during December 10-14, 1999, within a programme started some years ago and devoted to the optical polarization monitoring of $\mathrm{BL}$ Lac objects in both hemispheres, in a range of time scales from days down to less than an hour (Scaltriti et al. 1999; Treves et al. 1999; Tommasi et al. 2001b). 
A description of the observations and data reduction is given in Sect. 2; the results of linear and circular polarimetry are reported in Sect. 3 and discussed in Sect. 4. Preliminary and partial results were presented in Tommasi et al. (2001a).

\section{Observations}

We selected 11 northern BL Lacs among the brightest and most polarized objects observed by Impey \& Tapia (1988) and Takalo et al. (1992). During the first night of the run, we performed a brief photopolarimetric "survey" with short integration times on all the sources, to determine which of them were the brightest and most polarized at the epoch of observation, to be intensively monitored in the two following nights: $3 \mathrm{C}$ 66A and PKS $0735+178$ were chosen. The total effective integration times on these two objects were 4.75 and 5.5 hours, respectively. Five more BL Lacs included in the list were observed sparsely during the run: 1 Jy 0138-097 (only circular polarization), H 0414+009, PKS 0823-223, OJ 287, BL Lac. In Table 1 the list of the observed sources is reported along with some results and basic information (see following sections), together with three southern BL Lacs monitored by our team with the $2.15 \mathrm{~m}$ telescope of the Complejo Astronomico El Leoncito (CASLEO, Argentina; see Scaltriti et al. 1999; Tommasi et al. 2001a, 2001b; Treves et al. 1999).

The Turku polarimeter (Turpol) was used for the NOT observations. This is a double channel chopping photopolarimeter, designed by Piirola (1973). Although the instrument conception is rather old, it remains particularly suited to multifrequency observations, thanks to its capability of performing simultaneous photopolarimetry in five different bands. Polarization of the light collected by the telescope is determined by a half-wave or quarterwave retarder plate (to measure linear or circular polarization, respectively, with the highest efficiency), rotated through 8 positions by $22.5^{\circ}$ steps. Then a calcite slab splits the light into ordinary and extraordinary rays that pass through identical diaphragms. Both components of the diffuse background from adjacent sky patches enter both diaphragms, resulting in a cancellation of the polarization of sky light. Finally, the beam is split by four dichroic+bandpass filter combinations and sent to five photomultipliers. Each of these channels reproduces the spectral response of one of the $U B V R I$ Johnson-Cousins bands. In this way, truly simultaneous multiband observations can be performed. For all our observations, an integration time of $10 \mathrm{~s}$ was used for each position of the retarder plate, giving a polarimetric measurement every $3.5 \mathrm{~min}$. In order to improve the signal to noise ratio, intranight measurements were then binned with four plate cycles (about $15 \mathrm{~min}$ ). A $10 \mathrm{~s}$ sky background integration was normally performed every $15 \mathrm{~min}$. High and null polarization standard stars from Schmidt et al. (1992), observed several times per night, were used to determine the instrumental parameters: instrument induced polarization and orientation of the zero point of the retarder plate with respect to the North.

Data reduction was performed using dedicated routines. They allow calculation of polarization percentage $(P)$ and position angle (PA) by fitting the counts recorded in the eight positions of the retarder plate with a suitable cosine function using a least-squares algorithm. Subtraction of an average sky value obtained by interpolating between the nearest sky background acquisitions taken close to the object is automatically performed and the instrumental Stokes parameters are subtracted to obtain the true polarization state from the measured one. Error estimates take into account both the uncertainty from photon statistics and from the least-squares fit. In particular, the larger of these two contributions is adopted. In this way the error cannot be underestimated, as it could be if only the fit uncertainty was used. Binned points and nightly means can be calculated too, by averaging Stokes parameters over single polarimetric measurements.

A summary of the results is reported in Table 2 with $1 \sigma$ errors in brackets. The uncertainties for $3 \mathrm{C} 66 \mathrm{~A}$ and PKS 0735+178 during December 11 and 12 are the maximum between statistical errors associated with the individual points and standard deviations of intranight binned measurements. So, intrinsic variability of the objects during those nights is taken into account as a source of uncertainty on the nightly means.

Absolute flux measurements from the counts recorded by the photopolarimeter are much more sensitive than Stokes parameter measurements (involving differences between close count recordings) to sky background/transparency fluctuations and seeing effects, as well as to small pointing or guiding errors, especially if relatively low-brightness targets are observed. As a consequence, no useful simultaneous photometry could be extracted from our polarimetric data. Therefore, we used the best $V$-band images taken with the standby CCD camera (Stancam) during the pointing phase to each source to obtain information on the photometric state of the BL Lacs during our observations. Results are reported in Table 1. The magnitude differences measured between the standard stars included in the field of view are very similar to the standard $\Delta V$ values reported in the literature (Smith \& Sitko 1991; Fiorucci \& Tosti 1996; Fiorucci et al. 1998). Differential photometry yielded a precision of about $\pm 0.02 \mathrm{mag}$, but the $V$ magnitudes of the comparison stars are known with a greater error (about $\pm 0.06 \mathrm{mag}$ ). Hence, the uncertainties reported in brackets are mainly due to the reduction to the standard photometric system.

\section{Results}

\subsection{Linear polarization}

\subsubsection{C $66 \mathrm{~A}$}

Our results on this source, showing an average polarization of $27.3 \%$ in $U$ band, confirm that it is one of the most 
Table 1. Summary of the relevant parameters of the observed BL Lacs.

\begin{tabular}{lclccrrrrr}
\hline Object & Catalog, name & Type & \multicolumn{1}{c}{$z$} & \multicolumn{1}{c}{$m_{V}$} & $P_{m}^{a}$ & \multicolumn{1}{c}{$P_{\nu}^{b}$} & \multicolumn{1}{c}{$C D^{c}$} & $B^{d}$ & $\delta^{e}$ \\
\hline $0138-097$ & 1 Jy & LBL & 0.733 & - & - & - & no host & & \\
$0219+428$ & 1ES, 3C 66A & LBL & 0.444 & $14.80(0.05)$ & 26.2 & 0.10 & 36.3 & 0.59 & 15 \\
$0301-243^{f}$ & PKS & LBL & 0.260 & - & 8.2 & 0.54 & 7.7 & & \\
$0414+009$ & HEAO-A3 & HBL & 0.287 & $17.05(0.06)$ & 3.6 & - & 3.7 & & \\
$0735+178$ & PKS & LBL & $>0.424$ & $15.54(0.05)$ & 9.8 & 0.25 & $>35$ & 0.41 & 17 \\
$0823-223$ & PKS & LBL & $>0.910$ & - & 15.3 & 0.25 & no host & & \\
$0851+202$ & 1 Jy, OJ 287 & LBL & 0.306 & $16.72(0.09)$ & 12.4 & -0.28 & $>26$ & & \\
$2005-489^{f}$ & PKS & HBL & 0.071 & - & 4.3 & 0.31 & 5.2 & 1 & 15 \\
$2155-304^{f}$ & PKS & HBL & 0.116 & - & 4.7 & 0.42 & 7.2 & 1.22 & 18 \\
$2200+420$ & 1 Jy, BL Lac & LBL & 0.069 & $13.69(0.04)$ & 4.0 & -0.27 & 6.1 & 0.43 & 11 \\
\hline
\end{tabular}

${ }^{a}$ Average value of linear polarization in the $V$ band. For the objects observed in different epochs, the average absolute value of polarization percentage is given, neglecting the position angle.

${ }^{b}$ Wavelength dependence of polarization, as defined in Eq. (1). For objects observed in more than one night, the weighted average is given.

${ }^{c}$ Ratio between nuclear and galactic flux in $R$ band (Wurtz et al. 1996; Scarpa et al. 2000).

${ }^{d}$ Magnetic field intensity in Gauss (Ghisellini et al. 1998; Tagliaferri et al. 2001).

${ }^{e}$ Doppler factor (Ghisellini et al. 1998; Tagliaferri et al. 2001).

${ }^{f}$ Object observed by our team at the CASLEO telescope equipped with the Turin photopolarimeter (Scaltriti et al. 1999; Tommasi et al. 2001a, 2001b; Treves et al. 1999).
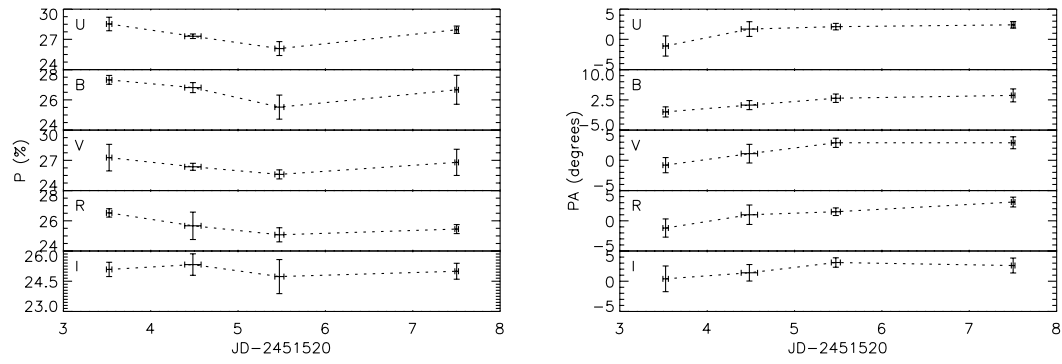

Fig. 1. Nightly mean values of $P$ (left panels) and PA (right panels) in $U B V R I$ bands for 3C $66 \mathrm{~A}$. Horizontal bars represent the duration of the observations.
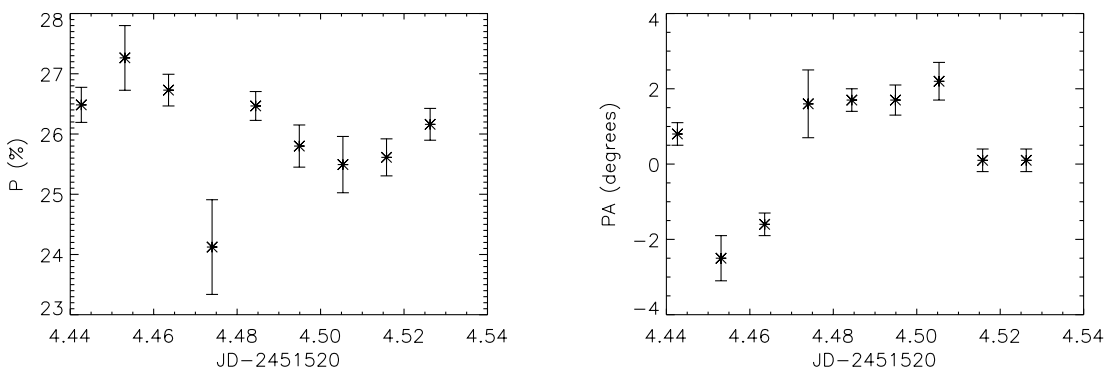

Fig. 2. Intranight behaviour of $3 \mathrm{C} 66 \mathrm{~A}$ during December 11, 1999 in $R$ band with 15 min time bins.

strongly polarized BL Lacs (Takalo \& Sillanpää 1993). Some day-to-day variability of both $P$ and PA is present (Fig. 1). A simple $\chi^{2}$ test shows significant variability of $P$ at a $95 \%$ confidence level or more in $U, B$ and $R$ filters and of $\mathrm{PA}$ in $U, V$ and $R$. Moreover, variations in the five bands are well correlated. In Fig. 2 polarization and position angle during the night of December 11 in the $R$ filter are reported, with 15 min binning. Variations are detected both in polarization and position angle, with higher significance for PA. The sharp minimum in PA is noticeably lasting for about half an hour in the first part of the night and recorded also in the other bands, while variations in $P$ are not significant in $U, B, V$ and $I$ filters.
A comparison of our results with the polarization measurements reported in the literature (Mead et al. 1990; Sitko et al. 1985; Smith et al. 1987; Takalo 1991; Takalo \& Sillanpää 1993; Takalo et al. 1994; Valtaoja et al. 1991) is given in Fig. 3. All the collected data are reported with the same weight, even if observations do not uniformly cover the spanned time period; most of them have been performed in short campaigns separated by long intervals during which the source was not observed. The $P$ values are rather scattered, and seem to concentrate in two broad regions of the plot: one between $10 \%$ and $18 \%$, the second between $25 \%$ and $31 \%$. However, the number of available measurements is too low to claim a real bimodal 

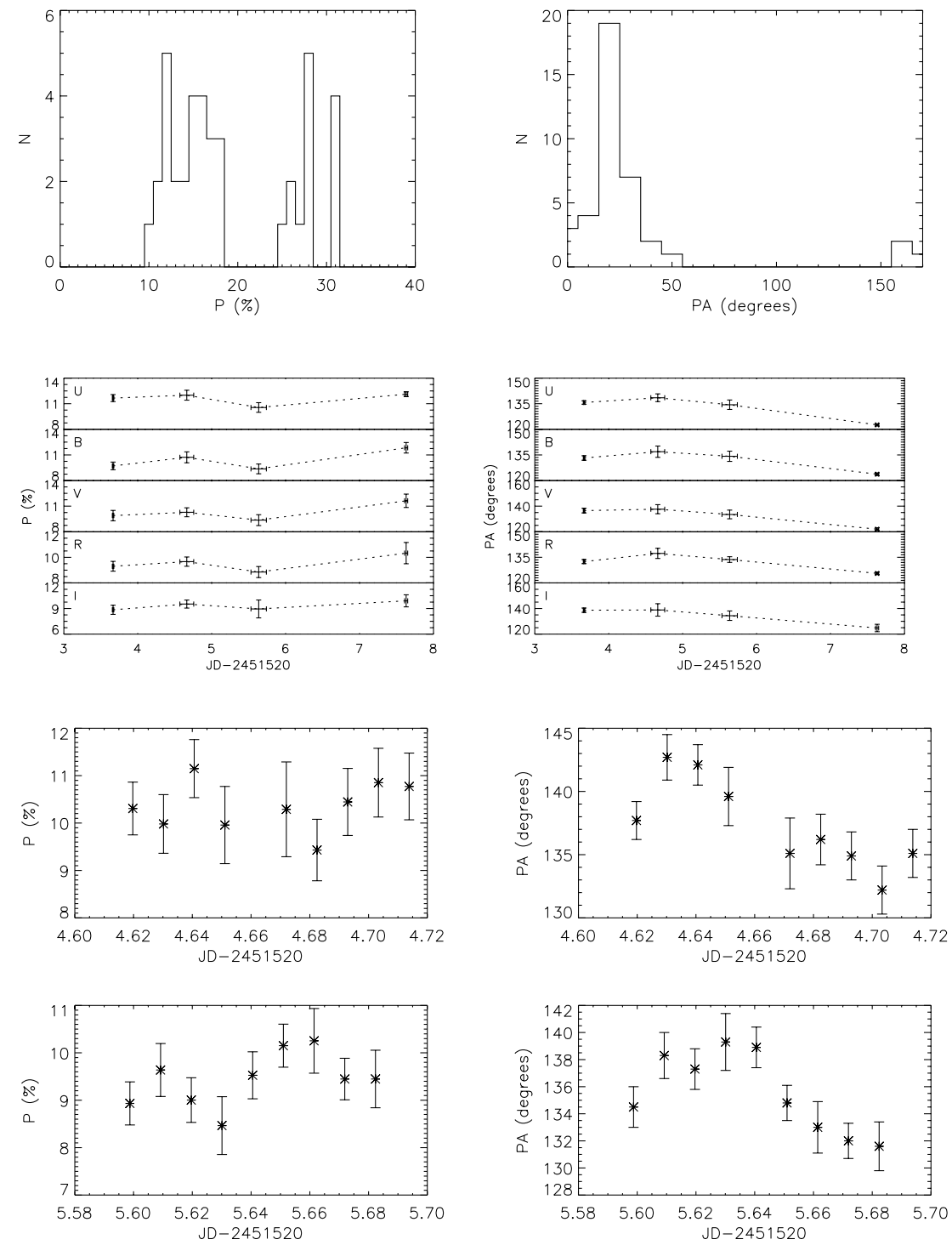

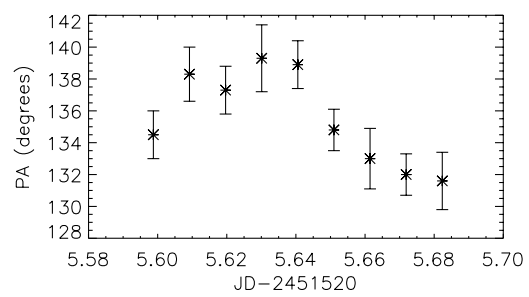

Fig. 3. Histograms of literature data for the $V$ band polarimetry of $3 \mathrm{C} 66 \mathrm{~A}$ (our results are included).

Fig. 4. Nightly mean values of $P$ (left panels) and PA (right panels) in $U B V R I$ bands for PKS $0735+178$. Horizontal bars represent the duration of the observations.

Fig. 5. Intranight behaviour of PKS 0735+178 during December 11 in $V$ band (upper panels) and December 12 in $B$ band with 15 min time bins. distribution. PA shows a strongly preferred value between $20^{\circ}$ and $30^{\circ}$. No correlation between $P$ and PA was found.

\subsubsection{PKS $0735+178$}

Nightly averages of $P$ and PA measurements for this source are reported in Fig. 4. Little variability is recorded in polarization percentage (significance level greater than $95 \%$ only in the $V$ filter), and more significant variations in PA are seen. In all bands, an increase in $P$ is seen in the last night, while the PA simultaneously rotates significantly with respect to the previous nights. Also on intranight time scales, more significant variations are seen in position angle than in polarization percentage. For the two nights with longest monitoring, we report in Fig. 5 the $P$ and PA curves in the filters where the variations are most apparent. Similar trends are seen also in the other bands.

Also for this BL Lac object, we searched the literature for previous polarimetric data (Brindle et al. 1986; Mead et al. 1990; Puschell et al. 1983; Sitko et al. 1985;
Smith et al. 1987; Takalo 1991; Takalo et al. 1992; Valtaoja et al. 1991, 1993) and reported them in histograms along with our data (Fig. 6) as done in the case of 3C 66A. Data are more sparsely distributed with respect to those of that object, but also in this case a broad peak in PA between $90^{\circ}$ and $160^{\circ}$ is suggested. The source showed very different levels of $P$ in the past years, covering the whole range from about $1 \%$ to more than $30 \%$.

\subsubsection{Other BL Lac objects}

Linear polarization measurements also were performed for four other BL Lac objects: H 0414+009, PKS 0823-223, OJ287 and BL Lac (see Table 2). PKS 0823-223 was observed twice and found to show different levels of polarization in December 10 and 14 . While $P$ was basically constant, PA rotated by about $3^{\circ}-8^{\circ}$ in the different filters. The other listed sources were observed sparsely during the run for short intervals, so no variability analysis could be performed. 

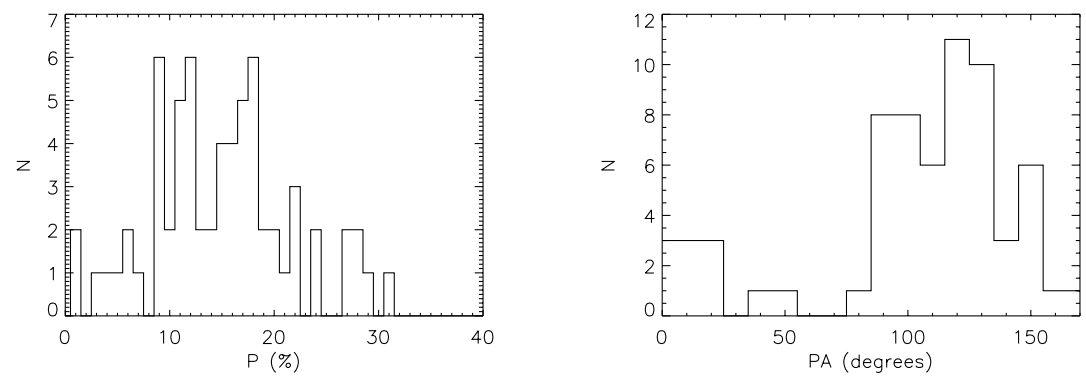

Fig. 6. Histograms of literature data for the $V$ band polarimetry of PKS 0735+178 (our results are included).

Comparison with literature data shows that the three sources H 0414+009 (Impey \& Tapia 1988; Mead et al. 1990), OJ 287 (e.g. Smith et al. 1987; Sillanpää et al. 1991; Takalo et al. 1992) and BL Lac (e.g. Puschell et al. 1983; Moore et al. 1987; Sillanpää et al. 1993) were found at polarization levels comparable to their historical values. In particular, the last two sources showed large variations in the past both in $P$ and PA and our values lie well within the observed variability ranges. PKS 0823-223 was found at a different polarization level with respect to that recorded at previous epochs. It has been observed in the past with $P$ ranging from $4.0 \%$ to $12.7 \%$ at wavelengths around $5000 \AA$ (Impey \& Tapia 1988; Wills et al. 1992; Visvanathan \& Wills 1998), while during our campaign it was polarized up to $15.6 \%$ in $V$ band. However, only few past measurements are available, so the level of polarization detected by us may be not that rare.

\subsection{Wavelength dependence of polarization}

Wavelength dependence of polarization (WDP) was investigated. Following Smith \& Sitko (1991), we introduced the parameters

$P_{\nu}=\frac{\mathrm{d} \log P}{\mathrm{~d} \log \nu}$

and

$\mathrm{PA}_{\nu}=\frac{\mathrm{dPA}}{\mathrm{d} \log \nu}$,

which describe the spectral shape of $P$ and PA. They were determined by fitting power-laws to the nightly means of the $U B V R I$ linear polarization percentages and position angles, considered as monochromatic measurements at the effective wavelength of the filters. In Table 3 we report the most statistically significant values of $P_{\nu}$ and $\mathrm{PA}_{\nu}$ obtained for some objects/nights, with their standard deviations resulting from the fit. In most cases $\mathrm{PA}_{\nu}$ is poorly determined and has been omitted in the table. H 0414+009 does not show significant wavelength dependence of polarization. While most objects show the usual trend with $P$ decreasing with decreasing frequency $\left(P_{\nu}>0\right)$, that can be partially due to dilution by the host galaxy light, noticeably two sources, namely BL Lac and OJ 287, show $P_{\nu}<0$. Similar results have been obtained for these two objects in past observations, see e.g. Sillanpää et al. (1991, 1993). For the sources observed
Table 3. Results from WDP analysis.

\begin{tabular}{ccccc}
\hline Object & $\begin{array}{c}\text { Date } \\
\text { (Dec. 99) }\end{array}$ & JD & $P_{\nu}$ & $\mathrm{PA}_{\nu}$ \\
\hline 3C 66A & 10 & 2451523 & $0.11 \pm 0.02$ & - \\
& 11 & 2451524 & $0.08 \pm 0.02$ & - \\
& 12 & 2451525 & $0.06 \pm 0.04$ & - \\
& 14 & 2451527 & $0.13 \pm 0.02$ & - \\
$0735+178$ & 10 & 2451523 & $0.29 \pm 0.06$ & - \\
& 11 & 2451524 & $0.26 \pm 0.07$ & - \\
& 12 & 2451525 & $0.21 \pm 0.09$ & - \\
& 14 & 2451527 & $0.21 \pm 0.06$ & $-3.6 \pm 1.5$ \\
$0823+223$ & 10 & 2451523 & $0.28 \pm 0.08$ & $5.6 \pm 2.4$ \\
& 14 & 2451527 & $0.24 \pm 0.04$ & $4.9 \pm 1.0$ \\
OJ 287 & 14 & 2451527 & $-0.28 \pm 0.08$ & - \\
BL Lac & 14 & 2451527 & $-0.27 \pm 0.05$ & $10.7 \pm 1.6$ \\
\hline
\end{tabular}

more than once during the campaign, no significant variability of the WDP can be evidenced.

\subsection{Circular polarization}

Circular polarization was investigated during the night of December 13, 1999 on four BL Lac objects (1 Jy 0138-097, 3C 66A, PKS 0735+178 and PKS 0823-223) and the results are reported in Table 2. Only in $3 \mathrm{C} 66 \mathrm{~A}$, the object with the smallest errors due to its relatively high brightness, was circular polarization marginally detected at the $2 \sigma$ level in $V$ and $R$ bands. A detection of non-null circular polarization in $3 \mathrm{C} 66 \mathrm{~A}$ was claimed by Takalo \& Sillanpää (1993), with larger values than found in our data. We conservatively put an upper limit of $0.4 \%$ with a $3 \sigma$ confidence level on the circular polarization of this BL Lac. In the case of PKS $0735+178$, marginal detection of circular polarization $P=-0.24 \% \pm 0.12 \%$ was reported by Valtaoja et al. (1993). However, no such polarization was recorded during our campaign, even if our uncertainty level is similar. Our data show an upper limit of about $0.4 \%$ at the $3 \sigma$ level for PKS $0735+178$. No significant circular polarization was found for the two other observed BL Lacs. Upper limits can be fixed at $2.5 \%$ and $0.5 \%$ for $1 \mathrm{Jy} 0138-097$ and PKS 0823-223, respectively. 


\section{Discussion}

The two sources which have been intensively monitored within this programme, 3C 66A and PKS 0735+178, exhibit a modest internight variability, which is more apparent in the position angle. This occurs also on an intranight scale and the overall behaviour is similar to that observed in B2 1308+326 (Puschell et al. 1979), BL Lac (Moore et al. 1987), S5 0716+174 (Impey et al. 2000) and PKS 2155-304 (Tommasi et al. 2001b). In the last three cases, total integration times were larger by an order of magnitude and more extreme variability episodes were recorded. As discussed by Tommasi et al. (2001b), a possible interpretation is that the emitting region is a relativistic jet, containing substructures of different polarization, with variable intensities.

Our results confirm the finding of a preferred polarization level over a time scale of years, indicating an underlying stability of the jet structure, even if in the presence of variability of the single subcomponents. In particular, the existence of a preferred position angle (see e.g. Jannuzi et al. 1994) could be related to a regular large-scale component of the magnetic field (Tommasi et al. 2001b).

A summary of the results of the present observations is reported in Table 1, together with data on some sources that have been observed previously by our team. We have also reported the core dominance $(C D)$ parameter in the $R$ band, i.e. the ratio between the nuclear intensity to that of the host galaxy, collected from the literature (Wurtz et al. 1996; Scarpa et al. 2000). It is apparent that the WDP is different for the various sources. Although the $P_{\nu}$ parameters should be corrected for dilution due to the host galaxy light (which in a first approximation can be considered as unpolarized), this difference holds also when comparing only the most core-dominated objects, for which the galaxy correction should be negligible. This may be ascribed to the different spectral appearance of LBL and HBL: with one exception (PKS 0301-243), the LBL in our sample have the lower values of $P_{\nu}$ (i.e., steeper polarized spectra) while the WDP for the HBL is flatter (although not significantly saw within the limited number statistics). This reflects the fact that, when the galaxy correction is negligible, the shape of the optical polarization should be that of "pure" synchrotron emission and therefore should depend on the position of the synchrotron peak (i.e., more HBL-like sources should have a flatter slope).

In two LBL in our sample, OJ 287 and BL Lac, the $P_{\nu}$ slope is negative, meaning that the polarization increases with wavelength. This is the opposite of what is expected for pure synchrotron radiation. Since the effect is significant, we argue that a source of thermal nature, like an accretion disk, may dilute the non-thermal emission of these objects and determine the observed wavelength dependence (see Wandel \& Urry 1991; Smith \& Sitko 1991). In BL Lac, an accretion disk has indeed been proposed by Corbett et al. (1996) and Corbett et al. (2000) as the powering source of the observed, variable $\mathrm{H} \alpha$ emission line. The presence of an accretion disk in OJ 287 has been

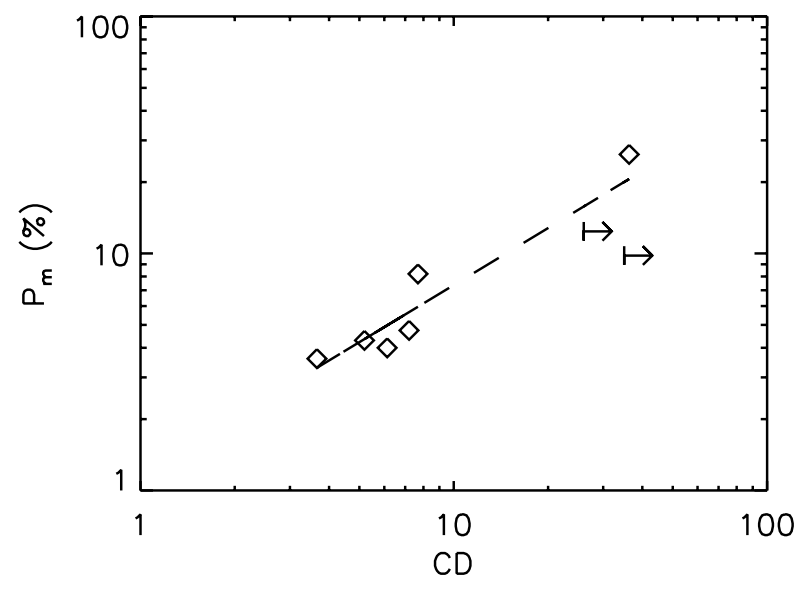

Fig. 7. Polarization vs. core dominance $(C D)$ for the objects in the sample. The dashed line represents the fit over the points with $C D \leq 10$, extrapolated towards higher values of $C D$. The arrows represent lower limits on the $C D$ for PKS $0735+178$ and OJ 287.

invoked to explain the observed flux and spectral variability (Sillanpää et al. 1988; Kidger et al. 1991; Katz 1997). However, the $P_{\nu}$ of OJ 287 in past polarimetric monitorings in optical bands has not been systematically negative (see e.g. Takalo et al. 1992), and, overall, the source exhibited preferentially a WDP behavior opposite to the one observed by us, namely decreasing polarization with increasing wavelength (Takalo 1994). One may argue that, since the accretion disk component is presumably more modestly variable than the beamed non-thermal one, it emerges during phases of quiescent synchrotron emission, so that a negative $P_{\nu}$ value may be more often seen during low brightness states of the source, such as the one we have monitored in our campaign.

Following Wurtz et al. (1996) we have plotted the average optical polarization in the $V$ band $P_{m}$ vs. $C D$ in Fig. 7. At the lower values, say $C D \leq 10$, there may be a monotonic growth, which could result only in part from the effect of dilution by the host galaxy. It has been highlighted in the figure by tracing the line representing a linear regression over those points in logarithmic scales. The fact that $3 \mathrm{C} 66 \mathrm{~A}$ (the object with the highest polarization recorded) lies on the line is noticeable and confirms that dilution is not the main cause of the observed dependence, since in this highly polarized and strongly core-dominated source, the host galaxy light should play little part. Even if the evidence of a $P$ vs. $C D$ correlation is based on few points, it interestingly confirms the suggestion of Wurtz et al. (1996), who found a correlation between two quantities at a $99 \%$ probability level. The result is not easy to interpret, and could suggest a correlation between polarization and beaming properties, which may manifest itself in the observed $C D$. A dependence of the observed polarization percentage from the degree of jet collimation and radiation beaming is expected, based on recent speculation made in the context of polarized afterglow emission from 
Gamma-Ray Bursts (Gruzinov 1999; Gruzinov \& Waxman 1999; Covino et al. 1999; Wijers et al. 1999). On the other hand, we note that the $C D$ parameter as defined here could reasonably be associated with the radio nuclear-toextended emission, which is a clear tracer of beaming in BL Lacs (see Fig. 5 in Ghisellini et al. 1993). Regrettably, we have insufficient statistics to prove the correlation of optical and radio $C D$ in our sample (only 4 of our objects have a measured radio $C D$ ), and the issue is in any case sensitive to nuclear variability, which is stronger in optical than in radio. We have therefore considered a model of the overall spectrum of blazars from radio to gamma ray frequencies, where a plasma radiates mainly via synchrotron and inverse Compton mechanisms. The relativistic motion of the plasma toward the observer is characterized by the beaming factor $\delta$. Our sample contains only BL Lac objects, and therefore the scattering of the relativistic electrons should occur mainly on the synchrotron photons, and the spectrum should be represented by the so-called synchrotron self-Compton model (SSC). The observation of the overall spectrum and of its time variability allows us to constrain the various parameters which enter the model (e.g. Ghisellini et al. 1998). In particular one can determine $\delta$ and the magnetic field intensity $B$, which a priori should correlate directly with polarization properties. Their values are reported in Table 1 (Ghisellini et al. 1998; Tagliaferri et al. 2001). Besides the well-known fact that LBL are more polarized than HBL (see e.g. Jannuzi et al. 1994; Ulrich et al. 1997), we notice that HBL appear to be associated with a stronger magnetic field in our sample. However, no clear direct dependence of $P$ on $\delta$ is apparent. Obviously, the objects under consideration are rather few. A search for a possible correlation of the circular polarization with $B$ or $\delta$ would be hazardous, because of the large uncertainties on $P$.

The investigation of polarization of BL Lac objects can give a large amount of information that could help to constrain jet models (Jones 1988; Björnsson 1993). Coordinated polarimetric monitoring at optical, nearinfrared and radio wavelengths has allowed several authors to elucidated the structure and distribution of the magnetic field, the interplay of different emission components and the location of emitting regions in blazars thanks to the variability of multiwavelength polarization percentage (e.g., Brindle et al. 1986; Kikuchi et al. 1988; Cawthorne \& Wardle 1988; Sillanpää 1991; Gabuzda \& Gómez 2001). Therefore other intensive campaigns seem strongly needed. In particular, simultaneous observations of the optical polarization and X-ray flux could better investigate the physical environment of the inner part of the synchrotron source. Moreover, simultaneous polarimetric and photometric data in the optical, best obtained with CCD-based instruments, could help to clarify how the injection of relativistic particles correlates with variations in the magnetic field.

Acknowledgements. We thank the NOT staff for technical support during the observations. Financial support from EC grant
ERBFMRXCT 98-0195 and Italian MURST COFIN 98021541 are acknowledged.

\section{References}

Allen, R. G., Smith, P. S., Angel, J. R. P., et al. 1993, ApJ, 403, 610

Björnsson, C.-I. 1993, ApJ, 416, 104

Brindle, C., Hough, J. H., Bailey, J. A., Axon, D. J., \& Hyland, A. R. 1986, MNRAS, 221, 739

Cawthorne, T. V., \& Wardle, J. F. C. 1988, ApJ, 332, 696

Corbett, E. A., Robinson, A., Axon, D. J., et al. 1996, MNRAS, 281,737

Corbett, E. A., Robinson, A., Axon, D. J., \& Hough, J. H. 2000, MNRAS, 311, 485

Covino, S., Lazzati, D., Ghisellini, G., et al. 1999, A\&A, 348, L1

Fiorucci, M., \& Tosti, G. 1996, A\&A, 116, 403

Fiorucci, M., Tosti, G., \& Rizzi, N. 1998, PASP, 110, 105

Gabuzda, D. C., Sitko, M. L., \& Smith, P. S. 1996, AJ, 112, 1877

Gabuzda, D. C., \& Gómez, J. L. 2001, MNRAS, 320, L49

Ghisellini, G., Padovani, P., Celotti, A., \& Maraschi, L. 1993, ApJ, 407, 65

Ghisellini, G., Celotti, A., Fossati, G., Maraschi, L., \& Comastri, A. 1998, MNRAS, 301, 451

Gruzinov, A. 1999, ApJ, 525, L29

Gruzinov, A., \& Waxman, E. 1999, ApJ, 511, 852

Impey, C. D., \& Tapia, S. 1988, ApJ, 333, 666

Impey, C. D., Bychkov, V., Tapia, S., Gnedin, Y., \& Pustilnik, S. 2000, AJ, 119, 1542

Jannuzi, B. T., Smith, P. S., \& Elston, R. 1994, ApJ, 428, 130

Jones, T. W. 1988, ApJ, 332, 678

Katz, J. I. 1997, ApJ, 478, 527

Kidger, M. R., Takalo, L. O., Valtaoja, E., de Diego, J. A., \& Sillanpää, A. 1991, A\&A, 252, 538

Kikuchi, S., Mikami, Y., Inoue, M., Tabara, H., \& Kato, T. 1988, A\&A, 190, L8

Mead, A. R. G., Ballard, K. R., Brand, P. W. J. L., et al. 1990, A\&AS, 83, 183

Moore, R. L., Schmidt, G. D., \& West, S. C. 1987, ApJ, 314, 176

Padovani, P., \& Giommi, P. 1995, ApJ, 444, 567

Piirola, V. 1973, A\&A, 27, 383

Puschell, J. J., Stein, W. A., Jones, T. W., et al. 1979, ApJ, 227, L11

Puschell, J. J., Jones, T. W., Phillips, A. T., et al. 1983, ApJ, 265,625

Saikia, D. J., \& Salter, C. J. 1988, A\&AR, 26, 93

Scaltriti, F., Raiteri, C. M., Villata, M., \& Anderlucci, E. 1999, in Blazar Monitoring towards the Third Millennium, Proceedings of the OJ-94 Annual Meeting 1999, ed. C. M. Raiteri, M. Villata, \& L. O. Takalo, Osservatorio Astronomico di Torino, Pino Torinese, Italy, 53

Scarpa, R., Urry, C. M., Falomo, R., Pesce, J. E., \& Treves, A. 2000, ApJ, 532, 740

Schmidt, G. D., Elston, R., \& Lupie, O. L. 1992, AJ, 104, 1563

Sillanpää, A., Haarala, S., Valtonen, M. J., Sundelius, B., \& Byrd, G. G. 1988, ApJ, 325, 628

Sillanpää, A. 1991, A\&A, 247, 11

Sillanpää, A., Takalo, L. O., Kikuchi, S., Kidger, M., \& De Diego, J. A. 1991, AJ, 101, 2017 
Sillanpää, A., Takalo, L. O., Nilsson, K., \& Kikuchi, S. 1993, Ap\&SS, 206, 55

Sitko, M. L., Schmidt, G. D., \& Stein, W. 1985, ApJS, 59, 323

Smith, P. S., \& Sitko, M. L. 1991, ApJ, 383, 580

Smith, P. S., Balonek, T. J., Elston, R., \& Heckert, P. A. 1987, ApJS, 64, 459

Tagliaferri, G., Ghisellini, G., Giommi, P., et al. 2001, A\&A, 368,38

Takalo, L. O. 1991, A\&AS, 90, 161

Takalo, L. O., \& Sillanpää, A. 1993, Ap\&SS, 206, 191

Takalo, L. O., Sillanpää, A., Nilsson, K., et al. 1992, A\&AS, 94,37

Takalo, L. O., Sillanpää, A., \& Nilsson, K. 1994, A\&AS, 107, 497

Takalo, L. O. 1994, Vistas Astron., 38, 77

Tommasi, L., Palazzi, E., Pian, E., et al. 2001a, Mem. S. A. It., in press
Tommasi, L., Díaz, R., Palazzi, E., et al. 2001b, ApJS, 132, 73

Treves, A., Pian, E., Palazzi, E., et al. 1999, Ap\&SS, 269, 667

Ulrich, M. H., Maraschi, L., \& Urry, C. M., 1997, ARA\&A, 35, 445

Valtaoja, L., Valtaoja, E., Shakhovskoy, N. M., Efimov, Yu. S., \& Sillanpää, A. 1991, AJ, 101, 78

Valtaoja, L., Karttunen, H., Valtaoja, E., Shakhovskoy, N. M., \& Efimov, Yu. S. 1993, A\&A, 273, 393

Visvanathan, N., \& Wills, B. J. 1998, AJ, 116, 2119

Wandel, A., \& Urry, C. M. 1991, ApJ, 367, 78

Wijers, R. A. M. J., Vreeswijk, P. M., \& Galama, T. J. 1999, ApJ, 523, L33

Wills, B. J., Wills, D., Breger, M., Antonucci, R. R. J., \& Barvainis, R. 1992, ApJ, 398, 454

Wurtz, R., Stocke, J. T., \& Yee, H. K. C. 1996, ApJS, 103, 109 